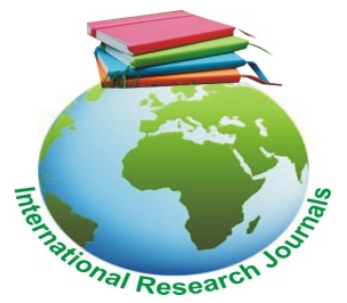

Educational Research (ISSN: 2141-5161) Vol. 7(3) pp. 055-071, May, 2016

Available online@ http://www.interesjournals.org/ER

DOI: http:/dx.doi.org/10.14303/er.2016.135

Copyright (C) 2016 International Research Journals

Full Length Research Paper

\title{
Influence of Physical Punishment Ban on Student Discipline in Public Secondary Schools in Kenya: A Case study of Ugenya, Gem and Siaya Sub-Counties
}

\author{
David Otieno Onyango and *Enose M.W. Simatwa \\ Department of Educational Management and Foundations, Maseno University \\ Department of Educational Management and Foundations, Maseno University \\ *Corresponding author's Email: simatwae@gmail.com
}

\begin{abstract}
The Kenya government outlawed Physical Punishment in schools through enactment of The Basic Education Act (2013). This was as a result of the recommendations of the Task Force on Student Discipline and Unrests in Secondary Schools which revealed that Physical Punishment was one of the major contributors to indiscipline among students. Despite the ban the level of indiscipline in schools had remained a major concern in Ugenya, Gem and Siaya sub-counties, where cases of indiscipline for the years 2010-2015 were 514 (51\%) higher than those experienced in Siaya County, 694 (44.1\%) and national, 22050 (42.7\%) for the same period. Therefore the objective of the study was to establish the influence of Physical Punishment ban on the level of student discipline in secondary schools in Ugenya, Gem and Siaya sub- counties. Discipline and organization among students in schools is vital in creating a conducive environment for learning. Discipline is a big concern for the teacher because the success or failure of a teacher or a principal of a school depends on it. Therefore students discipline is a critical factor in judging the performance of teacher. Besides, teachers are in loco parentis while a child is in school; they serve as surrogate parents. It is on this basis that teachers in Kenya were legally permitted to use physical punishment in 1972 through Legal Notice No. 40 of 1972. However, in 2001, the same Legal Notice was overturned by Legal Notice No. 56 of 2001, Children Act 2001, Constitution of Kenya, 2010 and The Basic Education Act 2013 because the disadvantages of use of physical punishment outweighed its advantage. It was also against the universal Declaration of Human Rights, that no person should be subjected to degrading treatment, torture and inhuman treatment, a product of United Nation to which Kenya is a signatory. It was also ostensibly against Kenya's penal code. The study established that physical punishment ban in secondary schools was about $60 \%$. The relationship between the physical punishment and level of student discipline was high, positive and statistically significant. Regression analysis revealed that increase in the increased level of student discipline in public secondary schools. Physical punishment means inflicting pain to a person's body. The forms of physical punishment include: canning, slapping, pinching, manual work, sitting in hot sun, kneeling, punching, running around school, play grounds, smacking, fetching water from streams, and spanking. The infractions to which these forms of physical punishment were found applied included: violence upon teachers and other fellow students, robbery, engaging in habitual profanity, vulgarity, sexual assault, terrorist threat against school authority extortion, death threats, drug abuse, vandalism, theft, disobedience, sneaking from school, truancy, possession of inflammable substances, firearms, gay, physical attacks, fighting, sexual harassment, bullying, noise making, unrest, strikes, fornication and flirtation. Prior to the ban the level of student discipline had seriously deteriorated to the extent that some parents were withdrawing their children from schools, which had been perceived earlier as havens of peace and institutions of character building apart from talent development and expertise re-engineering.
\end{abstract}

Keywords: Influence, Physical Punishment Ban, Student Discipline, Public Secondary Schools, Ugenya, Gem and Siaya Sub-Counties, Kenya.

\section{INTRODUCTION}

Students discipline is critical in attainment of positive school outcomes. This is because inter alia it provides a sense of direction among learners besides increasing a teacher's job satisfaction which is a critical correlate of 
commitment to school goals (Simiyu, 2003). In spite of the crucial role that discipline plays in the overall school outcomes, the condition of student discipline in Kenya's secondary schools has been disheartening. This is because hardly a school term goes without incidence of violent behavior being reported in the mass media (Ogetange, 2012).Teachers use different methods to manage discipline in schools, one of the methods used and the most controversial is physical punishment. In Kenya the government enacted The Basic Education Act, 2013, Republic of Kenya, 2013 which banned physical punishment in schools.

Mophosa and Shumba (2010) study revealed that the thrust of children's rights and subsequent banning of corporal punishment has ushered in an era of freedom for learners who no longer have respect or fear for their educators. The study also revealed that learners do not fear or respect educators because they know that nothing will happen to them. After the introduction of Free Primary Education and Free Secondary Education policies in Kenya, enrolment has increased tremendously resulting in shortage of teachers. The banning of corporal punishment compounded with high enrolment and shortage of teachers has resulted in increased indiscipline in schools. Discipline in Kenyan secondary schools is a matter of concern and the upsurge of indiscipline is blamed on the law that has in recent years forced teachers and even parents to spare the rod (Ogemba, 2011). A study by Kiprop (2004) confirms this, establishing that the banning of the cane has undermined discipline in schools and that discipline in secondary schools in Kenya in the post-caning era has deteriorated. According to a report by the Provincial Students' Discipline Committee in Central Province, indiscipline in secondary schools took various forms (Republic of Kenya, 2001a). Bullying was cited in this report to be one of the most common forms of indiscipline in secondary schools. The reviewed studies investigated the general level of discipline in schools after corporal punishment ban. The studies did not investigate the actual level of discipline in schools after physical punishment and mental harassment ban. This is the gap the current study strived to fill.

The Basic Education Act, 2013, (Republic of Kenya, 2013) does not give details of physical punishment. It is upon the individual teacher to interpret the Act. According to this study physical punishment includes caning, kneeling, pinching, manual work and any method of punishment that will inflict pain or involve physical torture of the student. Alternative methods of student discipline management include guidance and counseling, time off, withdrawal of privileges and suspension. Teachers are of the opinion that these alternative methods of student discipline management are not effective as they take too much time (Busienei, 2012). The government emphasized on guidance and counseling, as an alternative to physical punishment. According to Kaburu (2006) the use of guidance and counseling to manage student discipline is not effective because teachers lack guidance and counseling skills. This method is also time consuming and schools lack resources for effective guidance and counseling programs. Although the government has done a lot in order to curb violence and indiscipline in schools, there are still some cases of violence/strikes in schools. Furthermore many cases of other forms of indiscipline have been reported in the mass media (Murithi, 2010). Schools are now experiencing many forms of indiscipline as a result of physical punishment ban (Masau, 2012). Table 1 shows some forms of indiscipline cases experienced in Ugenya, Gem and Siaya Sub- Counties.

Records at Siaya county education office show that the number of reported indiscipline cases in the subcounties is higher than the national average level (Table 2).

In 2006 the United Nations Convention on the Rights of the Child resolved that children should be protected from physical punishment and non-physical forms of punishment that are cruel and degrading and thus incompatible with the convention. As a signatory to the convention, the Kenyan government therefore banned physical punishment and mental harassment in schools as stipulated in The Basic Education Act, 2013 (Republic of Kenya, 2013). Furthermore the Wangai report (Republic of Kenya, 2001a) also recommended the use of guidance and counseling instead of physical punishment and mental harassment. Records at the Siaya County Education Office show that from 2010 to 2015 the number of reported cases of students physical abuse by teachers was higher in Ugenya, Gem and Siaya Sub- Counties one hundred and twenty six out of one hundred and sixty eight (75\%) than the Siaya County schools average one hundred and forty two out of two hundred and sixty two (54\%)].These figures imply that level of physical punishment and mental harassment use in schools within Ugenya, Gem and Siaya Sub- Counties is higher than the Siaya County level and the national level.

This study therefore investigated the influence of physical punishment and mental harassment ban on student discipline in secondary schools in Ugenya, Gem and Siaya Sub- Counties, Kenya.

\section{Research Hypothesis}

$\mathrm{Ho}_{1}$ : There is no statistically significant relationship between physical punishment ban and the level of student discipline in public secondary schools. 
Table 1: Reported cases of indiscipline experienced in secondary school in Ugenya, Gem and Siaya SubCounties: 2010-2015

\begin{tabular}{lccccccc}
\hline Type of Indiscipline & $\mathbf{2 0 1 0}$ & $\mathbf{2 0 1 1}$ & YEAR 2012 & $\mathbf{2 0 1 3}$ & $\mathbf{2 0 1 4}$ & $\mathbf{2 0 1 5}$ & Total \\
\hline strikes & 15 & 14 & 13 & 10 & 10 & 9 & $\mathbf{7 1}$ \\
Bullying & 14 & 15 & 18 & 14 & 15 & 13 & $\mathbf{8 9}$ \\
Drugs & 10 & 11 & 11 & 14 & 15 & 17 & $\mathbf{7 8}$ \\
Mobile phones & 7 & 10 & 14 & 19 & 25 & 28 & $\mathbf{1 0 3}$ \\
Cheating/exams & 17 & 18 & 20 & 14 & 20 & 25 & 119 \\
Pregnancy & 6 & 9 & 8 & 9 & 10 & 12 & $\mathbf{5 4}$ \\
\hline
\end{tabular}

Source: Siaya County Director of Education office, 2015

Table 2: Indiscipline Level in Schools in Ugenya, Gem and Siaya Sub-Counties compared to Siaya County and National levels (20102015)

\begin{tabular}{|c|c|c|c|c|c|c|c|}
\hline $\begin{array}{l}\text { Forms of } \\
\text { indiscipline }\end{array}$ & Freq (f) & $\%$ age & $\begin{array}{c}\text { Siaya } \\
\text { County (f) }\end{array}$ & $\begin{array}{c}\text { Siaya } \\
\text { County(\%) }\end{array}$ & $\begin{array}{l}\text { National level } \\
\text { (f) }\end{array}$ & $\begin{array}{l}\text { National } \\
\text { level (\%) }\end{array}$ & $\begin{array}{l}\text { Gravity of } \\
\text { the offence }\end{array}$ \\
\hline Strikes & $71 / 168$ & 42.1 & $63 / 262$ & 24.2 & $1918 / 8600$ & 22.3 & $\begin{array}{l}\text {-Loss of property } \\
\text {-injury }\end{array}$ \\
\hline Bullying & $89 / 168$ & 53.2 & $111 / 262$ & 42.4 & $3457 / 8600$ & 40.2 & $\begin{array}{l}\text {-Mental torture } \\
\text {-physical injury }\end{array}$ \\
\hline Drug abuse & 78/168 & 46.4 & $100 / 262$ & 38.2 & $2864 / 8600$ & 33.3 & $\begin{array}{l}\text {-Poor performance } \\
\text {-drop out }\end{array}$ \\
\hline Phones & $103 / 168$ & 61.3 & $157 / 262$ & 60.1 & $5177 / 8600$ & 60.2 & $\begin{array}{l}\text {-Confiscation } \\
\text {-truancy }\end{array}$ \\
\hline $\begin{array}{l}\text { Cheating / } \\
\text { examinations }\end{array}$ & $119 / 168$ & 70.6 & $184 / 262$ & 70.1 & $6020 / 8600$ & 70.0 & $\begin{array}{l}\text {-Unrealistic results-results } \\
\text { withheld }\end{array}$ \\
\hline Pregnancy & $54 / 168$ & 32.2 & $79 / 262$ & 30.3 & $2614 / 5160$ & 30.4 & $\begin{array}{l}\text {-drop out } \\
\text {-repetition }\end{array}$ \\
\hline Total & $514 / 1008$ & $51 \%$ & $694 / 1572$ & $44.1 \%$ & $22050 / 51600$ & $42.7 \%$ & \\
\hline
\end{tabular}

\section{Synthesis of literature on influence of physical punishment ban on student discipline in secondary schools}

Although corporal punishment was abolished in many states in America, teachers still use it to manage student discipline in schools (Thomson; 2002, Smith, 2008). This shows that corporal punishment ban has not been fully implemented in schools in America. This is because teachers feel that corporal punishment is effective in managing student discipline in schools and its ban results in increase in indiscipline (Hornsby, 2003). Despite the fact that corporal punishment was abolished in Australia, teachers still use it as a last resort to manage student discipline (Brister, 1999).

Current research shows that cases of learner indiscipline are on the increase in South African schools and in some cases; learners are alleged to have murdered others in school premises (Zulu, Merwe and Walt, 2004). As such, a lot of learner indiscipline cases have been reported in schools and this has raised concerns about the safety of schools and classroom environments. In their study, Zulu et al (2004) reported cases of learner indiscipline in high schools in northern Durban. In a similar vein Aziza (2001) reported a sharp rise in cases of learners suspended and expelled from the Western Cape schools. Such cases of learner indiscipline have impacted negatively on teaching and learning in the schools (Zulu et al, 2004). Cases of learners injured and killed within the confines of the school are on the increase in South African schools. Press reports continue to alert the public on the rise of indiscipline cases (Thompson, 2002). The magnitude of reported cases of learner indiscipline warrants the use of different kinds of punishment-based disciplinary measures and the question still remains on the usefulness of such measures in curbing future occurrences of indiscipline or in helping the perpetrators (Thompson, 2002).

Simatwa (2007) carried out a study on management of student discipline in secondary schools in Bungoma County. The study employed survey design. Instruments of data collection were questionnaires, interviews and document analysis guide. The data collected was 
Independent variables

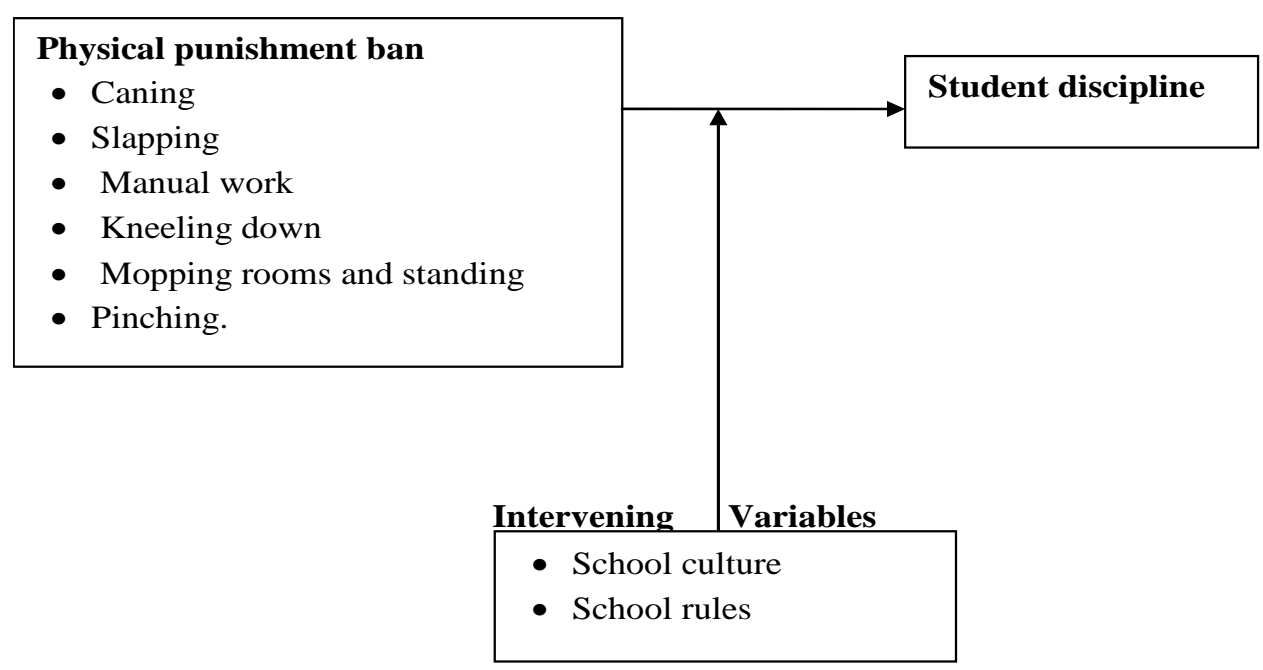

Figure 1: Conceptual Framework showing the influence of physical punishment ban students discipline

analyzed using descriptive statistics. The study revealed that teachers used a wide range of 'methods to manage student discipline. Teachers used methods like caning, kneeling, pinching, physical punishment, detention, reprimand, and exclusion among others. The findings of the study show that teachers use physical punishment like caning, kneeling and Physical Punishment to manage student discipline; especially for serious offences.

Busienei (2012) investigated the alternative methods which teachers used instead of corporal punishment and their efficacy. Instruments of data collection were questionnaires and interviews. The research study employed descriptive survey design. Data was analyzed using descriptive statistics. He found that, although teachers use alternative methods to corporal punishment, they believe that they are less effective compared to corporal punishment. In view of the findings, the study recommended urgent need to create awareness on alternative methods to corporal punishment and also on the overall effects of corporal punishment on the child. While the Busienei (2012) study focused on alternative methods which teachers used instead of corporal punishment and their efficacy, Simatwa (2007) study focused on management of student discipline in secondary schools. The present study was different in that it focused on the influence of physical punishment ban on student discipline in secondary schools. Both Simatwa (2007) study and Busienei (2012) study employed descriptive survey design. The current study employed both descriptive survey and correlational designs. While Busienei (2012) and Simatwa (2007) studies analyzed data using descriptive statistics, the current study analyzed data using both descriptive and inferential statistics. The reviewed studies investigated the implementation of corporal punishment ban in schools. But the studies did not investigate the effect of physical punishment ban on student discipline in secondary schools. The current study therefore attempted to fill this gap by investigating the influence of physical punishment ban on student discipline in secondary schools.

\section{Conceptual framework}

The study adopted a conceptual framework based on Douglas Mc Gregory's Theory "Y" (Owens, 1987) Figure 1. The conceptual framework (Figure 1) was based on the concept that when physical punishment is withdrawn and a conducive environment created are disciplined. people work. Coercion/ punishment is not needed for students to operate orderly and productively. The study investigated the influence of physical punishment ban on the level of student discipline in secondary schools. The conceptual framework postulates that physical

punishment ban is the independent variable that affects the level of student discipline which is the dependent variable. Independent variable is a variable that researchers manipulate in order to determine its effects on the dependant variable. The conceptual framework envisages that the independent variable physical punishment ban determine the level of student discipline 
in schools. From the literature reviewed physical punishment ban is more effective in student discipline management compared to alternative methods like guidance and counseling. Students prefer physical punishment ban resulting in high level of discipline. The use of alternative methods of discipline management is less effective and results in high levels of offences (Busienei, 2012). Intervening variable is one that moderates the independent variables influence on the dependent variable (Kenya Institute of Management, 2009). The conceptual framework postulates that intervening variables include school rules and school culture. For teachers to manage discipline using any discipline management method, there must be school rules in place. The school rules moderate the teachers influence as they manage student discipline without using physical punishment. School culture moderates discipline management methods in a school. Teachers' attitudes towards methods of discipline management will determine whether these methods will be effective or not. Teachers are the implementers of policies at the school level (Ouma et al, 2013). Discipline management methods can only have an effect on student discipline level only if they are fully implemented. As a consequent to physical punishment ban in schools teachers feel that they have been completely deprived of their powers and have no control over their students and they feel they have been given no alternatives. As a result they feel completely helpless (Kopansky, 2002). A study by Omboto (2013) found out that teachers had a negative attitude towards physical punishment ban in schools. The attitude of teachers towards government policies on discipline affects discipline management in schools since teachers are the implementers of policies at the school level. The government emphasizes on guidance and counseling for discipline management in schools. Teachers argue that they lack guidance and counseling skills. They therefore need in-service training in guidance and counseling for them to be able to use it effectively to manage student discipline. Teachers argue that alternative methods of discipline management like guidance and counseling take a lot of time which should be used for learning activities. They argue that such methods are only effective in schools where students have self discipline (Samoei, 2012).

School culture determines whether physical punishment can be used effectively to maintain discipline in schools. In some schools physical punishment is part of the school culture and students accept it. Physical punishment means methods that involve infliction of pain like caning and Physical Punishment. For example schools where the head teacher uses democratic style of management is likely to be inclusive whereby all stakeholders are involved and hence guidance and counseling method will be effective in discipline management. Suspension and expulsion are used as a last resort where other methods have failed. They are also used in cases of intolerable offences like fighting and destruction of property (Omboto, 2013).

\section{RESEARCH METHODOLOGY}

The study was guided by Douglas McGregor's Theory $Y$ (Owen's 1987). The study employed descriptive survey and correlational research designs. The study population was 10,629 subjects. That is, 168 principals, 168 deputy principals, 168 guidance and counseling teachers, 924 class representatives, 9200 form four students, and the Siaya County Director of Education. Stratified random sampling technique was used to select 116 principals, 116 deputy principals, 116 guidance and counseling teachers, 274 class representatives and 400 form four students. Saturated sampling was used to select the County Director of Education. The instruments of data collection were questionnaires, observation guide, interview schedule, focus group discussion guide, and document analysis guide. The content validity of the questionnaires, document analysis guide and interview schedule was addressed by research experts and their comments and suggestions were incorporated in the instruments. To enhance reliability, piloting was done in nine schools. Test-retest method was used to estimate the reliability of the instruments. Quantitative data on level of use of Physical Punishment and level of student discipline collected by use of questionnaires and document analysis guide was analyzed using frequency counts, percentages and means. Regression analysis was used to establish the influence of Physical Punishment ban on student discipline. Qualitative data collected by use of the in-depth interview, observation guide and focus group discussion was transcribed and arranged into themes and sub-themes as they emerged from the data.

\section{RESULTS}

\section{Demographic Characteristics of the Respondents and School Data}

Information on demographic characteristics of deputy principals, guidance and counseling teachers and students was obtained through questionnaires and document analysis. The information was tabulated as shown in Tables 3, 4 and 5 respectively. Table 3 shows that $50(43 \%)$ deputy principals were females while 66 (57\%) were males. Table 4 also shows that 39 (34\%) deputy principals had experience of 1-11 months, 49 (42\%) had experience of $1-2$ years and $28(24 \%)$ had 
060 Educ. Res.

Table 3: Deputy Principals' Demographic Characteristics

\begin{tabular}{lll}
\hline Demographic Data & Frequency $(\mathbf{f})$ & Percentage (\%) \\
\hline Gender & & \\
Female & 50 & 43 \\
Male & 66 & 57 \\
Total & 116 & 100 \\
Deputy headship experience & & \\
1-11 months & 39 & 34 \\
1-2 years & 49 & 42 \\
3-5 years & 28 & 24 \\
Total & 116 & 100 \\
\hline
\end{tabular}

Table 4: Demographic Characteristics of Guidance and Counseling Teachers'

\begin{tabular}{lcc}
\hline Demographic Characteristics & Frequency (f) & Percentage (\%) \\
\hline Gender & & \\
Female & 56 & 48 \\
Male & 60 & 52 \\
Total & $\mathbf{1 1 6}$ & $\mathbf{1 0 0}$ \\
Experience in Guidance \& Counseling & & \\
1-11 months & 50 & 43 \\
1-2 years & 38 & 33 \\
3-5 years & 28 & 24 \\
Total & $\mathbf{1 1 6}$ & $\mathbf{1 0 0}$ \\
\hline
\end{tabular}

Table 5: Demographic Characteristics of Class Representatives

\begin{tabular}{lcc}
\hline Demographic Data & Frequency (f) & Percentage (\%) \\
\hline Gender & & \\
Male & 152 & 56 \\
Female & 120 & 44 \\
Total Performance & $\mathbf{2 7 2}$ & $\mathbf{1 0 0}$ \\
& & \\
Above Average & 139 & 51 \\
Below Average & 133 & 49 \\
Total & $\mathbf{2 7 2}$ & $\mathbf{1 0 0}$ \\
\hline
\end{tabular}

experience of 3-5 years as deputy principals. Table 4 shows Guidance and Counseling teachers demographic characteristics.

Fifty six (48\%) guidance and counseling teachers were females while 60(52\%) were males. Table 4 also shows that $50(43 \%)$ guidance and counseling teachers had experience of 1-11 months as guidance and counseling teachers and $38(33 \%)$ had experience of $1-2$ years while $28(24 \%)$ had experience of 3-5 years. Table
4 shows class representatives demographic characteristics

Table 5 shows that 139 (51\%) students were above average while $133(49 \%)$ were below average in academic performance. Hence one hundred and fifty two (56\%) students were males and 120 (44\%) were females. Large student populations are more difficult to control than smaller populations. The schools were therefore categorized according to size. Table 6 shows that 51 
Table 6: Schools Data

\begin{tabular}{lcc}
\hline Category of school & Frequency $(\mathrm{f})$ & Percentage \% \\
\hline Single-stream & 51 & 44 \\
Multi- stream & 65 & 56 \\
Total & $\mathbf{1 1 6}$ & $\mathbf{1 0 0}$ \\
\hline
\end{tabular}

Table 7: Level of Student Discipline as Rated by Deputy Principals, Guidance and Counseling Teachers and Students

\begin{tabular}{|c|c|c|c|c|c|c|c|c|c|c|}
\hline \multirow{2}{*}{$\begin{array}{l}\text { Indicators } \\
\text { of discipline }\end{array}$} & \multirow[t]{2}{*}{ Resp } & \multicolumn{5}{|c|}{ Level of Discipline } & \multirow[t]{2}{*}{ MR } & \multirow[t]{2}{*}{ SD } & \multirow[t]{2}{*}{ OMR } & \multirow[t]{2}{*}{ ANOVA } \\
\hline & & VH & $\mathbf{H}$ & $\mathbf{M}$ & $\mathbf{L}$ & VL & & & & \\
\hline \multirow[t]{3}{*}{ Vandalism } & $\mathrm{D} / \mathrm{P}$ & 4 & 21 & 21 & 44 & 26 & 2.42 & 1.128 & 2.38 & $(F(2,501)=0.365, p=0.694)$ \\
\hline & $\mathrm{G} / \mathrm{C}$ & 3 & 21 & 21 & 44 & 27 & 2.39 & 1.109 & & \\
\hline & $\mathrm{S}$ & 8 & 44 & 44 & 108 & 68 & 2.32 & 1.106 & & \\
\hline \multirow[t]{3}{*}{ Noise making } & $\mathrm{D} / \mathrm{P}$ & 2 & 11 & 18 & 53 & 32 & 2.12 & 0.979 & 2.14 & $(F(2,501)=0.056, p=0.945)$ \\
\hline & $\mathrm{G} / \mathrm{C}$ & 3 & 12 & 19 & 49 & 33 & 2.16 & 1.038 & & \\
\hline & $\mathrm{S}$ & 5 & 27 & 44 & 120 & 76 & 2.14 & 0.994 & & \\
\hline \multirow[t]{3}{*}{ Lateness } & $\mathrm{D} / \mathrm{P}$ & 10 & 20 & 22 & 40 & 21 & 2.59 & 1.238 & 2.56 & $(F(2,501)=0.070, p=0.933)$ \\
\hline & $\mathrm{G} / \mathrm{C}$ & 13 & 12 & 20 & 49 & 25 & 2.53 & 1.233 & & \\
\hline & $\mathrm{S}$ & 27 & 38 & 49 & 104 & 54 & 2.56 & 1.235 & & \\
\hline \multirow{3}{*}{$\begin{array}{l}\text { Not doing } \\
\text { homework }\end{array}$} & $\mathrm{D} / \mathrm{P}$ & 6 & 20 & 23 & 45 & 22 & 2.51 & 1.138 & 2.51 & $(F(2,501)=0.333, p=0.717)$ \\
\hline & $\mathrm{G} / \mathrm{C}$ & 6 & 22 & 23 & 45 & 20 & 2.56 & 1.137 & & \\
\hline & $\mathrm{S}$ & 14 & 44 & 49 & 111 & 54 & 2.46 & 1.132 & & \\
\hline \multirow{3}{*}{$\begin{array}{l}\text { Sleeping in } \\
\text { class }\end{array}$} & $\mathrm{D} / \mathrm{P}$ & 3 & 21 & 20 & 35 & 37 & 2.29 & 1.172 & 2.32 & $(F(2,501)=0.117, p=0.890)$ \\
\hline & $\mathrm{G} / \mathrm{C}$ & 2 & 21 & 26 & 35 & 32 & 2.36 & 1.122 & & \\
\hline & $\mathrm{S}$ & 5 & 49 & 54 & 82 & 82 & 2.33 & 1.137 & & \\
\hline Not putting on & $\mathrm{D} / \mathrm{P}$ & 5 & 13 & 24 & 38 & 35 & 2.25 & 1.141 & 2.36 & $(F(2,501)=4.069, p=0.018)$ \\
\hline School & $\mathrm{G} / \mathrm{C}$ & 4 & 24 & 13 & 36 & 40 & 2.30 & 1.230 & & \\
\hline uniform & $\mathrm{S}$ & 11 & 44 & 44 & 87 & 86 & 2.29 & 0.971 & & \\
\hline Vulgar & $\mathrm{D} / \mathrm{P}$ & 13 & 20 & 20 & 41 & 22 & 2.66 & 1.278 & 2.57 & $(F(2,501)=0.524, p=0.593)$ \\
\hline \multirow[t]{2}{*}{ Language } & $\mathrm{G} / \mathrm{C}$ & 10 & 15 & 26 & 41 & 24 & 2.53 & 1.205 & & \\
\hline & $\mathrm{S}$ & 27 & 38 & 49 & 95 & 63 & 2.53 & 1.262 & & \\
\hline \multirow{3}{*}{$\begin{array}{l}\text { Vernacular } \\
\text { speaking }\end{array}$} & $\mathrm{D} / \mathrm{P}$ & 14 & 22 & 25 & 30 & 25 & 2.74 & 1.320 & 2.70 & $(F(2,501)=0.591, p=0.554)$ \\
\hline & $\mathrm{G} / \mathrm{C}$ & 14 & 20 & 26 & 35 & 21 & 2.75 & 1.278 & & \\
\hline & $\mathrm{S}$ & 27 & 44 & 60 & 81 & 60 & 2.62 & 1.265 & & \\
\hline Deviant & $\mathrm{D} / \mathrm{P}$ & 5 & 20 & 24 & 41 & 25 & 2.46 & 1.145 & 2.43 & $(F(2,501)=0.099, p=0.905)$ \\
\hline \multirow[t]{2}{*}{ behaviour } & $\mathrm{G} / \mathrm{C}$ & 4 & 22 & 22 & 41 & 26 & 2.44 & 1.144 & & \\
\hline & $\mathrm{S}$ & 11 & 44 & 54 & 98 & 65 & 2.40 & 1.136 & & \\
\hline \multirow[t]{3}{*}{ Sneaking } & $\mathrm{D} / \mathrm{P}$ & 4 & 20 & 12 & 52 & 28 & 2.32 & 1.124 & 2.26 & $(F(2,501)=0.368, p=0.692)$ \\
\hline & $\mathrm{G} / \mathrm{C}$ & 3 & 13 & 20 & 48 & 32 & 2.22 & 1.149 & & \\
\hline & $\mathrm{S}$ & 8 & 38 & 38 & 117 & 71 & 2.25 & 1.081 & & \\
\hline Boy-girl canal & $\mathrm{D} / \mathrm{P}$ & 6 & 21 & 23 & 45 & 21 & 2.53 & 1.138 & 2.56 & $(F(2,501)=0.562, p=0.571)$ \\
\hline \multirow[t]{2}{*}{ Knowledge } & $\mathrm{G} / \mathrm{C}$ & 6 & 21 & 23 & 45 & 21 & 2.53 & 1.138 & & \\
\hline & $\mathrm{S}$ & 16 & 54 & 68 & 85 & 49 & 2.51 & 1.160 & & \\
\hline Fighting in & $\mathrm{D} / \mathrm{P}$ & 6 & 30 & 34 & 26 & 20 & 2.79 & 1.161 & 2.85 & $(F(2,501)=0.320, p=0.726)$ \\
\hline \multirow{2}{*}{ school } & $\mathrm{G} / \mathrm{C}$ & 6 & 35 & 35 & 23 & 17 & 2.91 & 1.139 & & \\
\hline & $\mathrm{S}$ & 14 & 76 & 81 & 57 & 44 & 2.85 & 1.151 & & \\
\hline \multirow[t]{3}{*}{ Delinquency } & $\mathrm{D} / \mathrm{P}$ & 7 & 10 & 21 & 56 & 22 & 2.34 & 1.072 & 2.37 & $(F(2,501)=0.120, p=0.887)$ \\
\hline & $\mathrm{G} / \mathrm{C}$ & 7 & 13 & 21 & 55 & 20 & 2.41 & 1.088 & & \\
\hline & $\mathrm{S}$ & 16 & 27 & 49 & 131 & 49 & 2.20 & 1.073 & & \\
\hline Cheating in & $\mathrm{D} / \mathrm{P}$ & 3 & 12 & 15 & 44 & 42 & 2.05 & 1.070 & 2.03 & $(F(2,501)=0.064, p=0.938)$ \\
\hline \multirow[t]{2}{*}{ examinations } & $\mathrm{G} / \mathrm{C}$ & 2 & 11 & 17 & 42 & 44 & 2.02 & 1.034 & & \\
\hline & $\mathrm{S}$ & 5 & 27 & 33 & 109 & 98 & 2.02 & 1.024 & & \\
\hline Drug abuse & $\mathrm{D} / \mathrm{P}$ & 5 & 13 & 40 & 28 & 30 & 2.44 & 1.121 & 2.41 & $(F(2,501)=0.660, p=0.517)$ \\
\hline & $\mathrm{G} / \mathrm{C}$ & 4 & 15 & 39 & 30 & 28 & 2.46 & 1.099 & & \\
\hline & $\mathrm{S}$ & 8 & 27 & 82 & 87 & 68 & 2.34 & 1.050 & & \\
\hline
\end{tabular}


062 Educ. Res.

Table 7 continue

\begin{tabular}{lllllllllll} 
Bullying & $\mathrm{D} / \mathrm{P}$ & 4 & 12 & 45 & 37 & 18 & 2.54 & 0.990 & 2.54 & $(\mathrm{~F}(2,501)=0.002, \mathrm{p}=0.998)$ \\
School mates & $\mathrm{G} / \mathrm{C}$ & 5 & 11 & 44 & 37 & 19 & 2.53 & 1.017 & & \\
& $\mathrm{~S}$ & 11 & 27 & 103 & 87 & 44 & 2.54 & 1.009 & & \\
Theft in school & $\mathrm{D} / \mathrm{P}$ & 3 & 10 & 50 & 30 & 23 & 2.48 & 0.991 & 2.48 & $(\mathrm{~F}(2,501)=1.019, \mathrm{p}=0.362)$ \\
& $\mathrm{G} / \mathrm{C}$ & 2 & 9 & 47 & 35 & 23 & 2.41 & 0.952 & & \\
& $\mathrm{~S}$ & 5 & 27 & 121 & 81 & 38 & 2.42 & 0.915 & & \\
Disobedience & $\mathrm{D} / \mathrm{P}$ & 3 & 4 & 35 & 55 & 19 & 2.28 & 0.873 & 2.29 & $(\mathrm{~F}(2,501)=0.014, \mathrm{p}=0.986)$ \\
to teachers & $\mathrm{G} / \mathrm{C}$ & 2 & 5 & 35 & 56 & 18 & 2.28 & 0.842 & & \\
& $\mathrm{~S}$ & 5 & 16 & 88 & 109 & 54 & 2.30 & 0.915 & & \\
Truancy & $\mathrm{D} / \mathrm{P}$ & 2 & 5 & 23 & 55 & 31 & 2.07 & 0.892 & 2.10 & $(\mathrm{~F}(2,501)=0.213, \mathrm{p}=0.808)$ \\
& $\mathrm{G} / \mathrm{C}$ & 3 & 4 & 23 & 57 & 29 & 2.09 & 0.904 & & \\
Defiance & $\mathrm{S}$ & 5 & 16 & 60 & 120 & 71 & 2.14 & 0.932 & & \\
Curricular & $\mathrm{C} / \mathrm{P}$ & 3 & 11 & 20 & 55 & 27 & 2.21 & 0.991 & 2.25 & $(\mathrm{~F}(2,501)=0.475, \mathrm{p}=0.622)$ \\
activities) & $\mathrm{G} / \mathrm{C}$ & 2 & 12 & 22 & 56 & 24 & 2.24 & 0.957 & & \\
Overall & $\mathrm{S}$ & 8 & 33 & 54 & 117 & 60 & 2.31 & 0.038 & & \\
& $\mathrm{D} / \mathrm{P}$ & 5 & 16 & 26 & 43 & 26 & 2.41 & 1.128 & $\mathbf{2} .41$ & $\mathbf{( F ( 2 , 5 0 1 ) = 0 . 0 0 3 , p = 0 . 9 9 7 )}$ \\
& $\mathrm{G} / \mathrm{C}$ & 5 & 16 & 26 & 43 & 26 & 2.41 & 1.128 & & \\
& $\mathrm{~S}$ & 12 & 37 & 61 & 99 & 63 & 2.40 & 1.115 & & \\
\hline
\end{tabular}

Key: $\mathrm{VH}=$ =ery high, $\mathrm{H}=$ high, $\mathrm{M}=$ =moderate, $\mathrm{L}=$ low, $\mathrm{VL}=$ very low. Resp =respondents, $\mathrm{MR}=$ mean rate, $\mathrm{OMR}=$ overall mean rate, $\mathrm{SD}=$ standard deviation. The five point scale used was as illustrated:

\section{Interpretation of mean rating}

Mean ratings
$1.00-1.44$
$1.45-2.44$
$2.45-3.44$
$3.45-4.44$
$4.45-5.00$

$\begin{array}{lc}\text { Descriptor } & \text { Percentage } \\ \text { Very low } & 20 \\ \text { low } & 40 \\ \text { Moderate } & 60 \\ \text { High } & 80 \\ \text { Very high } & 100\end{array}$

(44\%) schools were single stream schools and 65 (56\%) schools were multi-stream schools.

\section{Objective}

The research objective was: To establish the influence of physical punishment ban on student discipline in secondary schools.

The hypothesis that was used to establish the influence of physical punishment ban on student discipline was: "There is no statistically significant relationship between physical punishment ban and students discipline in public secondary schools". The first step in data analysis involved descriptive analysis of the stress levels among students and the level of physical punishment ban in the three sub counties. Thus, to establish the influence of physical punishment ban on student discipline their level of student discipline was first established. The results were as shown Table 7.

From Table 7, it can be observed that the students level of discipline was mean rated at 2.41. This means that the student discipline level was low at about $40 \%$. The extent to which physical punishment ban was implemented in Ugenya, Gem and Siaya sub counties was established. The results were as shown in Table 8.

From Table 8 , it can be observed that the level of physical punishment ban in Ugenya, Gem and Siaya Sub-counties was mean rated at 2.88. This means that the level of physical punishment ban was at about $60 \%$ student discipline level was low at about $40 \%$. The data on the level of physical punishment ban and student level of discipline ban was correlated using Pearson Product Moment Correlation coefficient to determine the strength and direction of relationship so as to infer the influence of physical punishment ban on student discipline. The results were as shown in Table 9.

From Table 9 it can be noted that there was a strong positive and significant relationship between physical punishment ban and student discipline $(r=.879, N=504$, $\mathrm{p}<.05)$.

To estimate the influence of physical punishment ban on student discipline, coefficient of determination was computed. The results were as shown in Table 10.

From Table 10, it can be observed that physical punishment ban accounted for $77.3 \%$ of variation in student disciple line as signified by the Adjusted $\mathrm{R}$ square $\left(R^{2}\right) .773$. This implied that $77.3 \%$ of the total variance in 
Onyango and Simatwa, 063

Table 8: Extent of Physical Punishment Ban $(n=504)$

\begin{tabular}{|c|c|c|c|c|c|c|c|c|c|c|}
\hline \multirow[t]{3}{*}{ Offences } & \multirow[t]{3}{*}{ Res } & \multicolumn{5}{|c|}{ PHYSICAL } & \multirow[t]{3}{*}{ MR } & \multirow[t]{3}{*}{ SD } & \multirow[t]{3}{*}{ OMR } & \multirow[t]{3}{*}{ ANOVA } \\
\hline & & \multicolumn{3}{|c|}{ PUNISHMENT E } & \multicolumn{2}{|c|}{ Ban } & & & & \\
\hline & & VH & $\mathrm{H}$ & $M$ & $\mathbf{L}$ & VL & & & & \\
\hline \multirow[t]{3}{*}{ Truancy } & $\mathrm{D} / \mathrm{P}$ & 21 & 37 & 12 & 37 & 9 & 3.20 & 1.282 & 3.18 & $(F(2,501)=0.119, p=0.888)$ \\
\hline & $\mathrm{G} / \mathrm{C}$ & 21 & 37 & 11 & 37 & 10 & 3.19 & 1.298 & & \\
\hline & $\mathrm{S}$ & 46 & 85 & 27 & 90 & 24 & 3.14 & 1.288 & & \\
\hline \multirow[t]{3}{*}{ Noise } & $\mathrm{D} / \mathrm{P}$ & 11 & 18 & 35 & 35 & 17 & 2.75 & 1.282 & 2.22 & $(F(2,501)=0.103, p=0.902)$ \\
\hline & $\mathrm{G} / \mathrm{C}$ & 12 & 17 & 35 & 34 & 18 & 2.75 & 1.298 & & \\
\hline & $\mathrm{S}$ & 24 & 41 & 81 & 82 & 44 & 1.17 & 1.288 & & \\
\hline \multirow[t]{3}{*}{ Lateness } & $\mathrm{D} / \mathrm{P}$ & 11 & 23 & 23 & 44 & 15 & 2.75 & 1.171 & 2.75 & $(F(2,501)=0.174, p=0.840)$ \\
\hline & $\mathrm{G} / \mathrm{C}$ & 12 & 23 & 23 & 45 & 13 & 2.79 & 1.191 & & \\
\hline & $\mathrm{S}$ & 24 & 52 & 54 & 107 & 35 & 2.72 & 1.174 & & \\
\hline Not doing & $\mathrm{D} / \mathrm{P}$ & 18 & 37 & 21 & 29 & 11 & 3.19 & 1.244 & 3.14 & $(F(2,501)=0.406, p=0.666)$ \\
\hline \multirow[t]{2}{*}{ homework } & $\mathrm{G} / \mathrm{C}$ & 17 & 37 & 21 & 29 & 12 & 3.16 & 1.248 & & \\
\hline & $\mathrm{S}$ & 38 & 82 & 49 & 68 & 35 & 3.07 & 1.275 & & \\
\hline Sleeping in & $\mathrm{D} / \mathrm{P}$ & 21 & 35 & 26 & 23 & 11 & 3.28 & 1.241 & 3.25 & $(F(2,501)=0.156, p=0.856)$ \\
\hline \multirow{2}{*}{ class } & $\mathrm{G} / \mathrm{C}$ & 21 & 35 & 25 & 23 & 12 & 3.26 & 1.259 & & \\
\hline & $\mathrm{S}$ & 46 & 79 & 60 & 57 & 30 & 3.21 & 1.254 & & \\
\hline \multirow{3}{*}{ Improper dress } & $\mathrm{D} / \mathrm{P}$ & 23 & 21 & 45 & 18 & 9 & 3.27 & 1.170 & 3.24 & $(F(2,501)=0.263, p=0.769)$ \\
\hline & $\mathrm{G} / \mathrm{C}$ & 23 & 21 & 45 & 17 & 10 & 3.26 & 1.188 & & \\
\hline & $\mathrm{S}$ & 54 & 49 & 95 & 41 & 33 & 3.18 & 1.258 & & \\
\hline Vulgar Language & $\mathrm{D} / \mathrm{P}$ & 10 & 17 & 35 & 37 & 17 & 2.71 & 1.150 & 2.70 & $(F(2,501)=0.007, p=0.993)$ \\
\hline & $\mathrm{G} / \mathrm{C}$ & 9 & 18 & 35 & 36 & 18 & 2.69 & 1.145 & & \\
\hline & S & 22 & 41 & 82 & 86 & 41 & 2.70 & 1.145 & & \\
\hline Mother tongue & $\mathrm{D} / \mathrm{P}$ & 10 & 16 & 32 & 35 & 23 & 2.61 & 1.200 & 2.59 & $(F(2,501)=0.061, p=0.941)$ \\
\hline & $\mathrm{G} / \mathrm{C}$ & 9 & 16 & 33 & 35 & 23 & 2.59 & 1.165 & & \\
\hline & $\mathrm{S}$ & 22 & 38 & 71 & 81 & 60 & 2.57 & 1.204 & & \\
\hline Disruptive & $\mathrm{D} / \mathrm{P}$ & 11 & 18 & 27 & 37 & 23 & 2.63 & 1.234 & 2.61 & $(F(2,501)=0.141, p=0.868)$ \\
\hline & $\mathrm{G} / \mathrm{C}$ & 12 & 17 & 26 & 37 & 24 & 2.62 & 1.256 & & \\
\hline & $\mathrm{S}$ & 27 & 35 & 63 & 87 & 60 & 2.57 & 1.243 & & \\
\hline Sneaking & $\mathrm{D} / \mathrm{P}$ & 23 & 33 & 37 & 14 & 9 & 3.41 & 1.165 & 3.08 & $(F(2,501)=44.248, p=0.000)$ \\
\hline & $\mathrm{G} / \mathrm{C}$ & 23 & 32 & 37 & 14 & 10 & 3.38 & 1.184 & & \\
\hline & $\mathrm{S}$ & 54 & 76 & 82 & 33 & 27 & 2.46 & 1.051 & & \\
\hline $\mathrm{B} / \mathrm{G}$ relationship & $\mathrm{D} / \mathrm{P}$ & 23 & 33 & 39 & 12 & 9 & 3.42 & 1.151 & 3.10 & $(F(2,501)=45.933, p=0.000)$ \\
\hline & $\mathrm{G} / \mathrm{C}$ & 23 & 32 & 40 & 11 & 10 & 3.41 & 1.165 & & \\
\hline & $\mathrm{S}$ & 54 & 76 & 87 & 33 & 22 & 2.48 & 1.034 & & \\
\hline Disobedience & $\mathrm{D} / \mathrm{P}$ & 6 & 11 & 26 & 40 & 33 & 2.28 & 1.133 & 2.31 & $(F(2,501)=0.122, p=0.885)$ \\
\hline & $\mathrm{G} / \mathrm{C}$ & 6 & 12 & 25 & 41 & 32 & 2.30 & 1.136 & & \\
\hline & $\mathrm{S}$ & 14 & 27 & 73 & 82 & 76 & 2.34 & 1.138 & & \\
\hline Delinquency & $\mathrm{D} / \mathrm{P}$ & 6 & 11 & 35 & 35 & 29 & 2.40 & 1.118 & 2.41 & $(F(2,501)=0.022, p=0.978)$ \\
\hline & $\mathrm{G} / \mathrm{C}$ & 6 & 12 & 35 & 34 & 29 & 2.41 & 1.127 & & \\
\hline & $\mathrm{S}$ & 14 & 27 & 87 & 76 & 68 & 2.42 & 1.121 & & \\
\hline Cheating & $\mathrm{D} / \mathrm{P}$ & 11 & 33 & 49 & 11 & 12 & 3.16 & 1.100 & 3.19 & $(F(2,501)=0.265, p=0.768)$ \\
\hline & $\mathrm{G} / \mathrm{C}$ & 12 & 32 & 49 & 12 & 11 & 3.19 & 1.071 & & \\
\hline & $\mathrm{S}$ & 27 & 82 & 109 & 27 & 27 & 3.24 & 1.086 & & \\
\hline Drug abuse & $\mathrm{D} / \mathrm{P}$ & 18 & 29 & 48 & 11 & 10 & 3.29 & 1.111 & 3.29 & $(F(2,501)=0.088, p=0.916)$ \\
\hline & $\mathrm{G} / \mathrm{C}$ & 19 & 29 & 47 & 12 & 9 & 3.32 & 1.108 & & \\
\hline & $\mathrm{S}$ & 41 & 68 & 108 & 33 & 22 & 3.27 & 1.109 & & \\
\hline Bullying & $\mathrm{D} / \mathrm{P}$ & 6 & 11 & 32 & 35 & 32 & 2.34 & 1.135 & 2.33 & $(F(2,501)=0.030, p=0.971)$ \\
\hline & $\mathrm{G} / \mathrm{C}$ & 6 & 12 & 31 & 34 & 33 & 2.34 & 1.150 & & \\
\hline & $\mathrm{S}$ & 14 & 27 & 73 & 76 & 82 & 2.32 & 1.154 & & \\
\hline Theft & $\mathrm{D} / \mathrm{P}$ & 14 & 22 & 49 & 20 & 11 & 3.07 & 1.109 & 3.05 & $(F(2,501)=0.124, p=0.883)$ \\
\hline & $\mathrm{G} / \mathrm{C}$ & 14 & 22 & 49 & 19 & 12 & 3.07 & 1.109 & & \\
\hline & $\mathrm{S}$ & 33 & 52 & 108 & 46 & 33 & 3.02 & 1.157 & & \\
\hline Fighting & $\mathrm{D} / \mathrm{P}$ & 14 & 22 & 51 & 18 & 11 & 3.09 & 1.100 & 3.06 & $(F(2,501)=0.293, p=0.746)$ \\
\hline & $\mathrm{G} / \mathrm{C}$ & 14 & 22 & 51 & 17 & 12 & 3.08 & 1.112 & & \\
\hline & $\mathrm{S}$ & 33 & 52 & 108 & 41 & 38 & 3.00 & 1.179 & & \\
\hline
\end{tabular}


064 Educ. Res.

Table 8 Continue

\begin{tabular}{|c|c|c|c|c|c|c|c|c|c|c|}
\hline \multirow[t]{2}{*}{ Vandalism } & $\mathrm{D} / \mathrm{P}$ & 6 & 11 & 35 & 35 & 29 & 2.40 & 1.118 & \multirow[t]{2}{*}{2.40} & \multirow[t]{2}{*}{$(F(2,501)=0.608, p=0.992)$} \\
\hline & $\mathrm{G} / \mathrm{C}$ & 6 & 12 & 35 & 34 & 29 & 2.41 & 1.127 & & \\
\hline & $S$ & 14 & 27 & 81 & 82 & 68 & 2.40 & 1.119 & & \\
\hline \multirow[t]{3}{*}{ Defiance } & D/P & 11 & 18 & 27 & 37 & 23 & 2.63 & 1.234 & \multirow[t]{3}{*}{2.61} & \multirow[t]{3}{*}{$(F(2,501)=0.145, p=0.865)$} \\
\hline & $\mathrm{G} / \mathrm{C}$ & 12 & 17 & 26 & 37 & 24 & 2.62 & 1.256 & & \\
\hline & $\mathrm{S}$ & 24 & 38 & 63 & 90 & 57 & 2.57 & 1.216 & & \\
\hline \multirow{3}{*}{ Overall } & $\mathrm{D} / \mathrm{P}$ & 14 & 22 & 34 & 28 & 18 & 2.88 & 1.238 & \multirow{3}{*}{2.88} & \multirow{3}{*}{$(F(2,501)=0.065, p=0.937)$} \\
\hline & $\mathrm{G} / \mathrm{C}$ & 14 & 23 & 34 & 28 & 17 & 2.90 & 1.240 & & \\
\hline & $S$ & 31 & 53 & 78 & 66 & 44 & 2.86 & 1.235 & & \\
\hline
\end{tabular}

Key: $\mathrm{VH}=$ very high, $\mathrm{H}=$ high, $\mathrm{M}=$ moderate, $\mathrm{L}=$ low, $\mathrm{VL}=$ very low, $\mathrm{MR}=$ mean rate.

Resp=respondent, OMR=overall mean rate, $\mathrm{SD}=$ standard deviation, $\mathrm{S}=$ students,

D/P-=deputy principal, $\mathrm{G} / \mathrm{C}=$ guidance $\&$ counseling teachers. The five point scale used was as illustrated:

\section{Interpretation of mean rating}

$\begin{array}{lll}\text { Mean ratings } & \text { Descriptor } & \text { Percentage } \\ 1.00-1.44 & \text { Very low } & 20 \\ 1.45-2.44 & \text { low } & 40 \\ 2.45-3.44 & \text { Moderate } & 60 \\ 3.45-4.44 & \text { High } & 80 \\ 4.45-5.00 & \text { Very high } & 100\end{array}$

Table 9: Correlation analysis of the influence of Physical Punishment Ban and the Level of Student Discipline

\begin{tabular}{lll}
\hline & & Student discipline \\
physical punishment ban & Correlation & .879 \\
& Sig. (2-tailed) & .000 \\
& $\mathrm{~N}$ & 504 \\
\hline
\end{tabular}

Table 10: Regression analysis of the influence of Physical Punishment Ban and the Level of Student Discipline

\begin{tabular}{lcccc}
\hline Model & $\mathbf{R}$ & $\begin{array}{c}\mathbf{R} \\
\text { Square }\end{array}$ & $\begin{array}{l}\text { Adjusted } \\
\mathbf{R} \text { Square }\end{array}$ & $\begin{array}{c}\text { Std. } \\
\text { Error of the Estimate }\end{array}$ \\
\hline 1 & $.879^{\mathrm{a}}$ & .773 & .773 & .530 \\
\hline
\end{tabular}

a. Predictors: (Constant), physical punishment ban

Table 11: ANOVA of Physical Punishment ban and student discipline

\begin{tabular}{|c|c|c|c|c|c|c|}
\hline \multicolumn{2}{|c|}{ Model } & \multirow{2}{*}{$\begin{array}{l}\text { Sum of Squares } \\
480.201\end{array}$} & \multirow{2}{*}{$\begin{array}{l}\mathbf{d f} \\
1\end{array}$} & \multirow{2}{*}{$\begin{array}{l}\text { Mean Square } \\
480.201\end{array}$} & \multirow{2}{*}{$\begin{array}{l}\mathbf{F} \\
1.712 \mathrm{E} 3\end{array}$} & \multirow{2}{*}{$\begin{array}{l}\text { Sig. } \\
.000^{\mathrm{a}}\end{array}$} \\
\hline 1 & Regression & & & & & \\
\hline & Residual & 140.838 & 502 & .281 & & \\
\hline & Total & 621.040 & 503 & & & \\
\hline
\end{tabular}

a. Predictors: (Constant), physical punishment ban

b. Dependent Variable: level of student discipline

the level of student discipline was accounted for by physical punishment ban. The other $22.7 \%$ was due to other factors.
To establish whether physical punishment ban was a predictor of student discipline ANOVA was computed. The results were as shown in Table 11. 
Table 12: Simple Regression analysis of Physical Punishment Ban and Student Discipline

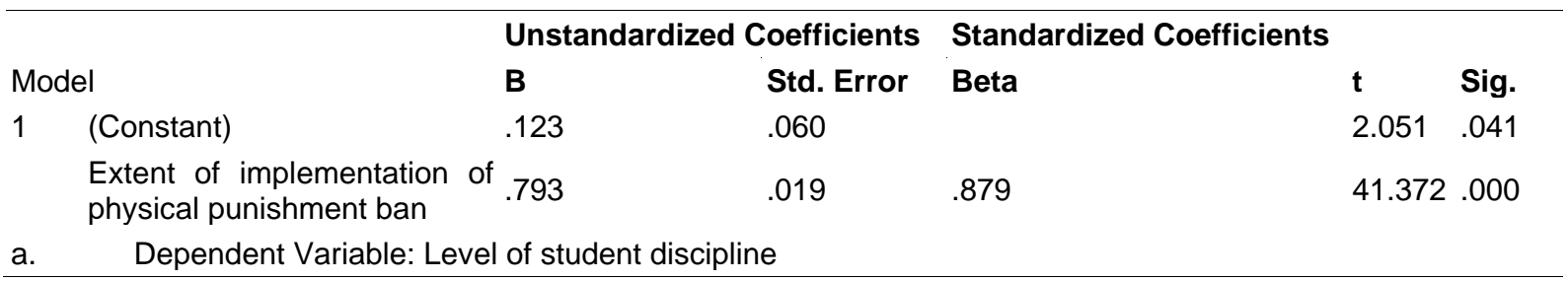

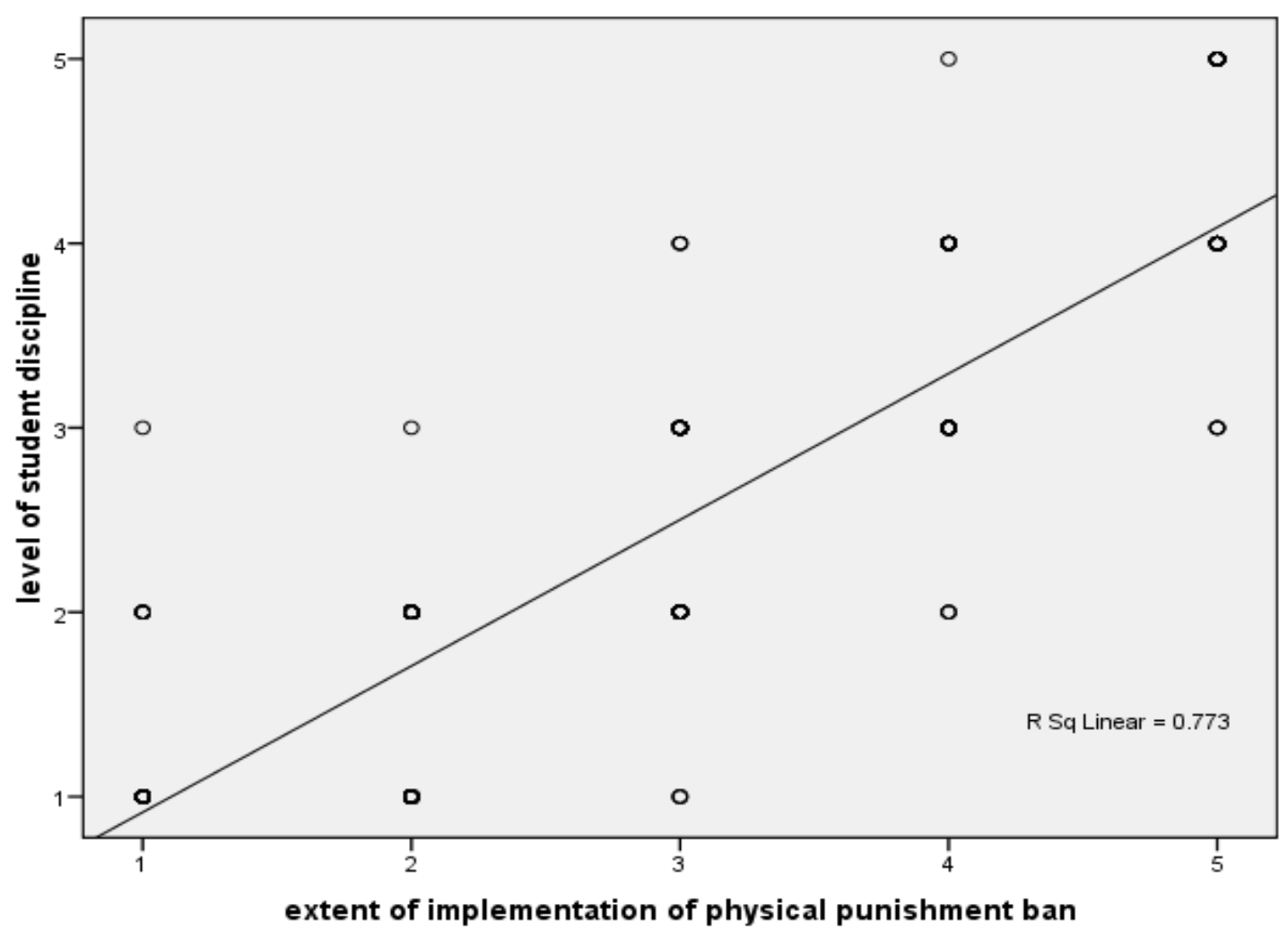

Figure 2: Scatter plot illustrating the relationship between physical punishment ban and student discipline

From Table 11, it can be noted that physical punishment ban was a predictor of student discipline $(F$ $(1,502)=583.502, p<.05)$.

To establish the actual influence physical punishment ban on student discipline simple regression was computed. The results were as shown in Table 12.

From Table 12 it can be noted that one unit increase in physical punishment ban can improve students discipline by .793 units. The regression equation is $\mathrm{Y}=$ $.123+.793 \mathrm{X}$
A scatter plot was used to illustrate the relationship between physical punishment ban and student discipline. (Figure 2)

The scatter plot indicates that there was a linear positive relationship between physical punishment ban and student discipline. That is, increase in implementation of physical punishment ban increases the level of student discipline. This means that if the ban is fully implemented the level of student discipline will be very high. 
066 Educ. Res.

\section{DISCUSSION}

Information was collected from both bright students and weak students. Students who perform poorly in examinations are often given physical punishment and in some cases reprimanded. Academically weak students are punished more often than bright students. This makes academically weak students to have a more negative attitude towards physical punishment and mental harassment. Male and female students perceive punishment differently (Mudemb, 2010). It was therefore necessary to collect information from both male and female students. Deputy principals, Guidance and counseling teachers and class representatives rated the extent of physical punishment ban implementation as moderate with respect to physical punishment use to control drug abuse by students. This indicates that physical punishment was moderately used to control drug abuse. Drug abuse is best dealt with by guidance and counseling rather than physical punishment (Gikonyo, 2002). Deputy principals, Guidance and counseling teachers and class representatives rated the extent of physical punishment ban implementation at moderate with respect to controlling sleeping in class. This indicates that physical punishment is moderately used to control sleeping in class. Students who sleep in class may be having family related problems. The class teacher should try to find out the root cause of this behavior (Egan, 2002). A student commended on sleeping in class: "Those students who sleep in class are either made to stand in class, sent out of class or told to write commitment letter. It all depends on the teacher." Standing in class is physical torture. Deputy principals, Guidance and counseling teachers and class representatives rated the extent of physical punishment ban at moderate with respect to controlling inappropriate dress code. This indicates that physical punishment is moderately used to control inappropriate dress code. Students may fail to put on the required attire because parents have not paid for it or it may have been stolen. Deputy principals, Guidance and counseling teachers and class representatives rated the extent of physical punishment ban at moderate with respect to controlling cheating in examinations. This indicates that physical punishment was moderately used to control cheating in examinations. Students cheat in examinations because of pressure from teachers and parents. The problem of students cheating in examination is best solved by the student being sent home to call the parents. The parents, together with the teachers and the students, jointly set achievable objectives for the student according to his ability. The ability of the student must be considered (Nyagah, 2008). Deputy principals, Guidance and counseling teachers and class representatives rated the physical punishment ban implementation at low with respect to controlling noise making. This indicates that physical punishment is highly used to control noise making. This finding is supported by (Ouma et al, 2013) who found out that teachers use physical punishment to control noise making. One student gave evidence to this when she said: "In this school water is a big problem. Those who commit some offences like noise making are told to go and bring water from the nearby stream to the kitchen."

Deputy principals, Guidance and counseling teachers and class representatives rated the extent of physical punishment ban as low with respect to bullying. This indicates that physical punishment was highly used to control bullying in schools. Deputy principals, Guidance and counseling teachers and class representatives rated the extent of physical punishment ban at low with respect to controlling disobedience in school. This indicates that physical punishment is highly used to control disobedience in schools. This finding is supported by Simatwa (2007) who found out that disobedience was one of the offences controlled by physical punishment. Deputy principals, Guidance and counseling teachers and class representatives rated extent of physical punishment ban in schools as moderate. Therefore physical punishment ban has not been fully implemented in schools. Teachers are still using physical punishment to manage discipline in schools. This finding is supported by a study carried out by Human Rights Watch (2005) which found that teachers still used corporal punishment to control student discipline in schools. The researcher observed students doing physical punishment in many schools. The study therefore established that physical punishment ban in Ugenya, Gem and Siaya sub-counties was low. This finding concurs with studies carried out by Thomson (2002), Smith (2008), Simatwa (2007) and Busienei (2012). These studies investigated the methods used by teachers to manage discipline in schools but they did not investigate the extent of physical punishment ban in schools. The current study established that physical punishment ban in schools in Siaya, Gem and Ugenya Sub Counties was moderate. Therefore this is the new knowledge generated by this study. During an interview with the principals one principal said that in large schools you cannot manage student discipline minus a cane. The director of education also seemed to confirm this when he said that his office has received many complaints about teachers using physical punishment to control student discipline. During the focus group discussion, students revealed that teachers used physical punishment to manage student discipline in schools. Surprisingly, school records like punishment books, did not show the use of physical punishment. This shows that the teachers intentionally avoided recording physical punishment probably because it is illegal. Evidence to this was given by one principal who said: 
"Right now you cannot see a cane here but it will appear immediately it is needed." This obviously indicates that the principals hide the canes from visitors.

Schools that have implemented physical punishment ban have high level of discipline. In these schools, teachers use alternative methods of discipline management like guidance and counseling (Busienei, 2012). Since these methods are learner friendly the students will cooperate with the teachers. The students will not rebel against methods that they find acceptable and pro-human rights. Furthermore, the guidance and counseling will make students to be self disciplined (Masitsa, 2008). These findings were supported by a principal who said that: "schools that have implemented physical punishment ban tend to have high level of discipline. In such schools teachers guide and reason with students and the students see the need for discipline. The students become self disciplined with subsequent increase in student discipline." Another principal put it that: "In large schools with high student population teachers claim guidance and counseling cannot work considering the high student population and understaffing problems. Students are therefore coerced to be disciplined using traditional methods like physical punishment to save on time. In the absence of the teachers the students become indisciplined. Sometimes students rebel against these traditional methods resulting in more indiscipline." The cited studies did not relate physical punishment ban and level of student discipline. The current study did establish that there is a high positive relationship (coefficient of correlation 0.879) between level of physical punishment ban and student discipline. This is the new knowledge generated by the study.

With the enactment of the Basic Education Act 2013, the government banned physical punishment and mental harassment and emphasized on guidance and counseling for discipline management in schools. For full implementation of physical punishment ban, teachers should be trained in guidance and counseling. Guidance and counseling helps to make students self disciplined as they see the need to be disciplined (Kaburu, 2006). A self disciplined student does not need to be coerced to behave well. All he needs is guidance. Hence no need for physical punishment and mental harassment. Although corporal punishment was abolished in many states in America, teachers still use it to manage student discipline in schools (Thomson, 2002, and Smith, 2006). This shows that corporal punishment ban has not been fully implemented in schools in America. This is because teachers feel that corporal punishment is effective in managing student discipline in schools and its ban will result in an increase in indiscipline (Hornsby, 2003). Despite the fact that corporal punishment was abolished in Australia, teachers still use it as a last resort to manage student discipline (Brister, 1999).

According to According to Thomson (2002), Smith (2006) and Brister (1999) Physical punishment is still used to control discipline in American and Australian schools. Hornsby (2003) found that teachers still use physical punishment to manage discipline in schools because it is effective and its ban will result in an increase in indiscipline. This is contrary to the findings of the current research study which found that physical punishment ban will result in an increase in student discipline. In South Africa, Mophosa and Shumba (2010) study revealed that the thrust of children's rights and subsequent banning of corporal punishment has ushered in an era of freedom for learners who no longer have respect or fear for their educators. They concluded that educators were aware of the need to protect children's rights and also ensuring that they were disciplined. However, educators felt that the alternative disciplinary measures to corporal punishment were not effective. Hence educators generally feel disempowered in their ability to institute discipline in schools in the absence of corporal punishment. The study also revealed that learners do not fear or respect educators because they know that nothing like Physical Punishment will be administered to them. This has resulted in an increase in indiscipline in schools. Current research shows that cases of learner indiscipline are on the increase in South African schools and in some cases; learners are alleged to have murdered others in school premises (Zulu, Merwe and Walt, 2004). As such, a lot of learner indiscipline cases have been reported in schools and this has raised concerns about the safety of schools and classroom environments.

Simatwa (2007) carried out a study on management of student discipline in secondary schools in Bungoma County. The study revealed that teachers used a wide range of 'methods to manage student discipline. The findings of the study showed that teachers used physical punishment like caning, kneeling and slapping to manage student discipline; especially for serious offences.

Busienei (2012) investigated the alternative methods which teachers used instead of corporal punishment and their efficacy. He found that, although teachers used alternative methods to corporal punishment, they believed that they are less effective compared to corporal punishment. In view of the findings, the study recommended urgent need to create awareness on alternative methods to corporal punishment and also on the overall effects of corporal punishment on the child. This recommendation is in line with the finding of the current study which found out that there is need to create awareness on physical punishment ban. If students have 
a positive attitude towards physical punishment ban the level of discipline will increase.

Studies by Smith (2006), Simatwa (2007) and Busienei (2012) show that physical punishment ban has not been fully implemented in schools. Teachers are still using physical punishment to manage student discipline in schools. Studies by Hornsby (2003), Mophosa and Shumba (2010) and Zulu et al (2004) reveal that the level of discipline in schools is low. These research findings are in line with the current research which found that low level of physical punishment ban will result in low level of discipline. Hence the high level of indiscipline in schools can be attributed to low level of physical punishment ban. During an interview, one principle made the following comments on the extent of physical punishment ban in schools and the level of student discipline: "We are required to use guidance and counseling to discipline students. Physical punishment is now illegal in schools. We have tried it here but it did not work. I hear it has worked in Europe and somebody somewhere felt that it can also be implemented in Africa. Let's wait and see. An African child is not like a European child. Conditions here are very different. Look at the understaffing here. How many teachers are trained in guidance and counseling? If teachers already are overworked, the syllabus not yet completed, books unmarked, where is the time for talking to individual students? Let alone talking to their parents. And do their parents come to school when they are called?" The comments made by the principal show that physical punishment ban has not been implemented in schools. The principal lamented that few teachers are trained in guidance and counseling. Furthermore teachers are overworked with heavy workloads that they do not have time for guiding and counseling individual students. Lack of guidance and counseling and the persistent use of physical punishment has resulted in low level of student discipline. This is in line with current study which found that low level of physical punishment will result in low level of discipline. The cited studies did not relate physical punishment ban and level of student discipline. The current study did establish that there is a high positive relationship $(r=.879, N=504, p<.05)$ between level of physical punishment ban and student discipline in Ugenya, Gem and Siaya Sub-counties. This is the new knowledge generated by the study. Figure 1 indicates that as physical punishment ban increases (becomes more positive) the level of students discipline increases. The study found that the overall physical punishment ban was $2.88(60 \%)$ which corresponds to moderate ban and the level of student discipline was 2.41 which corresponds to low level of discipline in Ugenya, Gem and Siaya- Sub counties. From Figure 4.4 the expectation is that moderate physical punishment ban would result in moderate level of discipline. But according to the findings of the study the level of student discipline was low. This discrepancy between the real results and the expected results can be explained by the fact that the physical punishment ban accounted for $77.3 \%$ of students level of discipline and other factors accounted for $22.7 \%$ hence the discrepancy.

\section{CONCLUSION}

The study concluded that the level of student discipline after the ban of physical punishment was low and the extent of physical punishment ban in schools in Ugenya, Gem and Siaya Counties were low and moderate with the overall mean ratings of 2.41 and 2.88 respectively. The study established that there was a strong relationship between physical punishment ban and the level of student discipline in secondary schools. Thus, the higher the extent of physical punishment ban the higher the level of student discipline. According to the findings of the current study, the low (2.41) level of student discipline in Ugenya, Gem and Siaya Sub-Counties was attributed to the moderate level (2.88) of physical punishment ban in managing student discipline. Student discipline level was low because physical punishment ban had not been fully implemented.

\section{RECOMMENDATIONS}

There is need for schools to implement physical punishment ban as stipulated in The Basic Education Act, 2013. The Ministry of Education Science and Technology should ensure that this policy is implemented in all schools. Quality and standards assurance officers should visit schools frequently to ensure that schools have implemented the ban.

Seminars and in-service courses should be organized for teachers to be enlightened on alternative approaches in managing student discipline.

The Ministry of Education, Science and Technology should ensure that quality assurance staff within the ministry visit schools frequently to guide teachers on implementation of physical punishment ban and also make follow up visits to schools.

In-service training and seminars should be organized for teachers to further train teachers in student discipline management techniques using the alternative methods.

Teacher training colleges and universities should train teachers on student discipline management using alternative methods. The curriculum should be reviewed to include this. The government should collect teachers' views on the problems and challenges they face in implementing physical punishment ban in schools and discuss with the teachers the solutions to these problems and the way forward. 
The Ministry of Education, Science and Technology should organize for bench marking trips where teachers would visit schools that have successfully implemented physical punishment ban. This will motivate teachers to try it in their respective schools.

\section{REFERENCES}

Aluru L (2013). "Four Maranda students held over school fire." Standard Digital. Nairobi: Nation Media Group p.1. February 2).

Afullo PTO (2007). Role of Teachers in the Management of Student Discipline in Public Schools in Bondo District Unpublished Master's thesis. Maseno University, Kenya.

Ajayi JA (2009). Parents Involvement in School Administration as a Correlate of Effectiveness of Secondary Schools in Nigeria. J. Educational Administration and Policy Studies, 18 (3), 41-46.

Ajowi, J.O. (2005). The Role of Guidance and Counseling in Promoting Student Discipline in Secondary Schools in Kisumu District. Unpublished Master's thesis. Maseno University, Kenya.

Aziza A (2001). Expulsion of Learners from Secondary Schools in Western Cape: Trends and Reasons. Unpublished Master's dissertation. Uni. South Africa.

Blandfords S (2000). Managing Discipline in Schools. London: Routlegde

Borg WR, Gall PJ, Gall DM (1993). APhysical Punishment lying Edu. Res.. New York: Longman

Brister C (1999). Corporal Punishment in Australian Schools. Available: url:http://www.nt/gov.au

Burgess RG (2000). In the Field. An Introduction to Field Res. London: George Allan

Busienei A (2012). Alternative Methods to Corporal Punishment and their Efficacy. J. of Emerging Trends in Educational Res. and Policy Studies, 3 (2), 155-161.

Butler B (1999). Problems of Childhood and Adolescence. London: Macmillan Press

Cheruiyot E (2001). A Study of Discipline Problems Affecting Kabianga Secondary School in Belgut Division, Kericho District (Unpublished P.G.D.E. Res. Project). Maseno Uni. Kenya.

Chumo D (2008). Effect of Withdrawal of Corporal Punishment on Primary School Pupils' Performance: A Case of Eldoret West District, Kenya. Master's thesis. Mount Kenya University

Cicognani L (2004). To Punish or Discipline? Teachers Attitudes towards the Abolition of Corporal Punishment Unpublished Master's thesis. Johannesburg Uni. South Africa.

Cohen L, Manion L (1994). Res. Methods in Edu. London and New York: Rutledge

Cotton K (2005). School wide and Classroom Discipline. Portland: Northeast Educational Laboratories

Daily Post $\left(2013\right.$, October $\left.16^{\text {th }}\right)$. 'Ambira high school closed indefinitely'. Daily Post. p.16.

Dietz TL (2000). Disciplining Children: Characteristics associated with the use of Corporal Punishment. Child Abuse and Neglect, 24(12), $1529-1542$

Daily Nation (2008, July $23^{\text {rd }}$ ). 'The government has banned the use of mobile phones in schools by students'. Daily Nation. Nairobi: Nation Media Group. p.3

Damien MM (2012). Attitudes of Stakeholders towards Physical Punishment on Pupils of International and National Schools in Kampala District, Uganda. World J. Edu, 2(1), 46-62.

Dewalt B (2002). Participant Observation: A Guide for Fieldworkers. Walnut Creek: Altmara Press

Duda FD (1996) Guidance and Counseling in Schools: A Case Study in Machakos Municipality. Unpublished P.G.D.E. Thesis. Kenyatta University.

Dowling E (2000). Theoretical Framework: A Joint Systems Physical Punishment roach to Educational Problems with
Rutledge

Egan E (2002). The Skilled Helper: Development of Physical Punishment roach to Helping. Chicago: Brook Cole

Elliot DS (1999). The Effect of Neighbourhood Discipline on Adolescence Development. J. Res. in Crime and Delinquency, 33(4), 389-426.

Fraenkel RJ, Wallen EM (2009). How to Design and Evaluate Research in Education. New York: Mac Graw Hill Inc.

Gay L (1996). Educational Res. Competence for Analysis and APhysical Punishment lication. New York: Macmillan

Gichuru J (2005). A Study on the Impact of the Ban on Corporal Punishment on Discipline of Students in Public Secondary Schools in Kikuyu Division, Kiambu District (Master's thesis). University of Nairobi, Kenya.

Gikonyo OM (2002). Drug abuses and Parental Knowledge of Drugs in Nairobi Province, Kenya (Master's thesis). Kenyatta University, Kenya.

Gitonga K (2011).'Police sent to school after riots'. Daily Nation, p.32. Nairobi; Nation Media Group September.

Gladwell A (1999). A Survey of Teachers' Attitudes towards Corporal Punishment after the Abolishment o Corporal Punishment (Master's Dissertation). University of Western Cape, Bellville.

Griffin G (2005). School Mastery: Straight Talk about Boarding School Management in Kenya. Nairobi: Lectern Publications.

Grinnel MJ (1993). Social Work Research and Evaluation. Illinois: Peacock Publishers

Harber C (2001). Schooling and Violence in South Africa: Creating a softer School. Intercultural Edu. 12(1), 261-271.

Hornsby G (2003). Teachers and Counseling. Routledge: Falmer

Human Rights Watch (2005). Spare the Child. Available: http://www.hrw.org

Imber M, Neidt WA (1999). Teacher Participation in School Decision Making. Newsbury Park: Sage Publication Inc

Indoshi FC (2003) "Teachers experiences of the Problem period of Teaching in Kenya: Implications for teacher Induction policies and Programs." J. of In- Service Edu. An Intl. J. Professional Develop. 29(3), 473-488.

Iqbal N (2003). Rights-Pakistan: Call to spare the rod in schools grows louder. New York: Global Information Network.

Kaburu LW (2006). Effectiveness of Guidance and Counseling Program in Combating Indiscipline in Secondary Schools: A Case of Nakuru Municipality, Kenya. Unpublished Master's thesis. Egerton University, Kenya.

Kariuki WE (2008). Factors that Lead to Secondary School Students Unrests in Kigumo District, Murang'a County (Master's thesis). Mount Kenya University, Kenya.

Kenya Institute of Management. (2009) Fundamentals of Management Research Methods Nairobi:Macmillan

Kerlinger FN (2007). Foundations of Behavioural Res. London: Holt and Winston

Kiarie J (2012). Indiscipline; Now Parents Perpetuate Vice. The Standard Online Available online http:// Digital std.org

Kilonzo J (2009). Challenges Faced by Head teachers in the Management of Students Indiscipline in Public Secondary Schools in Lamu County. Unpublished Master's thesis. Kenyatta University, Kenya.

Kindiki JN (2009).Effectiveness of Communication on Students Discipline in Secondary Schools in Kenya. Edu. Res. and Review, 4(5): 252- 259.

Kiprop CJ (2004). A Physical Punishment roaches to Management of Discipline in Secondary Schools in Kenya (Unpublished PhD thesis). Moi University, Kenya.

Kiptela C, Okero L, Kiprutu M (2011). Students Perceptions of Corporal Punishment in Taita Taveta County, Kenya (Unpublished Master's thesis). Egerton Uni. Kenya.

Kiumi JK (2008). Relationship between the Principals' Management $A$ Physical Punishment roaches and Level of Students' Discipline in Public Secondary Schools in Nyandarua and Laikipia Districts, Kenya (Unpublished PhD thesis). Egerton Uni. Kenya. 
Kopansky TM (2002). Corporal Punishment in Schools: A hit or Miss Proposition London: Longman

Kurui C (2012). Influence of School Management Strategies on Students Discipline: A Case of Secondary Schools in Mogotio District, Kenya (Unpublished Master's thesis). Mount Kenya University, Kenya.

Mac Donald MI (1997). Violence in Schools: Multiple Realities. J. of Edu. Res. 3(2), 142-155.

Masau KJ (2012). Emerging Issues as a Result of Banning Corporal Punishment as a Disciplinary Method in Primary Schools in Wote Division Makueni District (Unpublished P.G.D.E. Project). Kenyatta University, Kenya.

Masha J (2013). 'Survey reveals minors taking alcohol'. Digital Standard p.1, August19 Available: http.//std.news.org

Masitsa G (2008). Discipline and disciplinary measures in the Free State Township schools: Unresolved problems. Acta Academica, 40(2), 234-270

Maxwell WS (1999). Teachers Attitudes towards Disruptive Behavior in Secondary Schools. Educational Review, 39(3), 203-216.

Mbindyo MN (2006). Discipline Management and Control as Perceived by Students in Machakos District Kenya (Unpublished P.G.D.E. Thesis). Maseno University, Kenya.

Mbiti MD (2002). Foundations of School Administration. Nairobi: Oxford University Press

Migiro N (1996). An Investigation into the State of Guidance and Counseling in Secondary schools of Borabu Division in Nyamira District (Unpublished P.G.D.E. Research Project). Kenyatta University, Kenya.

Miriti MJ (2008). Challenges Facing School Administrators in Enforcing Students' Discipline in Public Secondary Schools in Yatta District, Machakos County (Master's thesis). Mount Kenya Uni. Kenya.

Mkangi K (2001). 'Characteristics that can lead to students' violence'. The Standard p.18. Nairobi: The Standard Group

Morrel R (2001). Corporal Punishment in South African Schools: A Neglected Explanation for its Persistence. South African J. of Edu. 21(3), 292-299. August 6

Mophosa C, Shumba A (2010). Educators' Disciplinary Capabilities After the Banning of Corporal Punishment in South African Schools. South African. Journal of Education, 30(2), 387-399.

Mosoti M (2011). 'Students charged with stealing laptops'. The Standard, p.4. October 13 Nairobi: The Standard Group

Mtsweni J (2008). The Role of Educators in the Management of School Discipline in the Nkangala region of Mpumalanga (Master's dissertation). University of South Africa.

Mudemb EV (2010). Causes of Drop out Among Boys and Girls from Secondary Schools in Ugenya District, Siaya County, Kenya (Unpublished Master's thesis). Uni. of Nairobi, Kenya.

Mugenda OM, Mugenda AG (2003). Research Methods: Quantitative and Qualitative APhysical Punishment roaches. Nairobi: Act Press

Murithi EW (2010). Challenges Principals Face in Enhancing Student Discipline in Secondary Schools in Tagania District, Kenya (Unpublished Master's thesis). Chuka University, Kenya.

Mutala JM (2008). Challenges Faced by Secondary School Students in Learning Mathematical Concepts in Makueni County, Kenya (Unpublished Master's thesis). Mount Kenya Uni. Kenya.

NACADA (2004). Youth in Peril. Nairobi: Government Printers

Naong M (2007). The Impact of abolition of Corporal Punishment on the Teacher Morale. South African J. Education, 27(2), 283- 300.

Ndiritu J (1996). An Investigation into the Provision of Guidance and Counseling Services in Secondary Schools in Gatundu South Division (Unpublished P.G.D.E. Project). Kenyatta Uni. Kenya.

Nduku MJ (2004). An investigation into Alternative Strategies of Discipline in the Absence of Corporal Punishment in the Public Secondary Schools in Matungulu Division, Machakos, District, Kenya (Unpublished Master's thesis). University of Nairobi, Kenya.

Nelson DL (2002). Organizational Behaviour: Foundations, Realities and Challenges. Canada: Thompson

Ng'ong'a BG (2002). "An Assessment of English Language Teachers

Edu. in the light of Classroom Needs: A case Study of Maseno Uni."
(Unpublished Doctoral Dissertation). Maseno Uni. Kenya

Njambi N (2008). Influence of Students' Discipline on Performance in K.S.C.E. in Nakuru Municipality, Kenya. (Unpublished Master's thesis). Mount Kenya Uni. Kenya.

Nyaga NE (2008). Influence of Students Discipline on Performance in K.C.S.E. in Public Secondary Schools in Nakuru Municipality, Kenya (Unpublished Master's thesis). Mount Kenya Uni. Kenya.

Ogemba P (2011). 'Students told to shun drugs'. Daily Nation, p. 9. November 18 Nairobi: Nation Media Group. Children. London:

Ogetange TB (2012).Teachers and Pupils' Views on Persistent use of Corporal Punishment in Managing Discipline in Primary Schools in Starehe Division, Kenya. Intl. J. Humanities and Soc. Sci, 19(2), 2342.

Oketch W (2011). 'Students sent home at night'. The Standard, p. 28 September 28.

Ombati C (2011). Students told to concentrate on their studies. The Standard, P.October 20

Ombiro, D. (2008). Challenges Facing Teachers in Enhancing Discipline among School Students in Kuria West, Migori County. Masters Thesis: Mount Kenya University.

Omboto JC (2013). Preparedness of Teachers to Maintain Discipline in the Absence of Corporal Punishment in Bondo District, Kenya (Unpublished Master's thesis). Maseno University, Kenya.

Omondi C (2010). The Level of Indiscipline after Banning Corporal Punishment in Schools. A Case Study of Ogande Girls High School in Rangwe Division, Homabay District (Unpublished P.G.D.E. Res. Project). Kenyatta Uni. Kenya.

Ouma MA, Simatwa EMW, Serem TDK (2013). Management of Pupil Discipline in Kenya: A Case Study of Kisumu Municipality. Available online@http://www. Interesjournals.org.

Owens RG (1987). Organizational Behaviour in Education. New Jersey: Prentice -Hall, Inc.

Owiti F (2001). "Experts give Causes of School Strikes". The Standard,. Nairobi: Standard Media Group. p. 17

Patton MO (1990). Qualitative Evaluation and Research Methods. Newbury Park, London: Sage

Republic of Kenya (1968). Education Act Chapter 212, (1968) Revised (1980) Laws of Kenya. Nairobi: Government Printers

Republic of Kenya (2001). Report of the Task Force on Students' Discipline and Unrest in Secondary Schools. Nairobi: Ministry of Education, Science and Technology.

Republic of Kenya (2001b). The Children's Act, Nairobi: Government Printer.

Republic of Kenya (2013). The Basic Education Act. Chapter 212. Nairobi: Government Printer.

Rosen L (1999). School Discipline: Best Practices for Administrators California: Corwin Press

Samoei WK (2012). The Role of Guidance and Counseling in Management of Student Discipline in Secondary Schools in Londiani District, Kericho County, Kenya (Unpublished Master's thesis). Kenyatta University, Kenya.

Sogoni N (1997). Attitudes of Students, Parents and Teachers towards the Use of Corporal Punishment in Senior Secondary Schools (Unpublished Master's thesis). University of Natal, Pietermaritzburg.

Simatwa EMW (2007). Management of Student Discipline in Secondary Schools in Bungoma District (Unpublished Doctor of Philosophy thesis). Maseno Uni. Kenya.

Simiyu CN (2003). Attitudes of Teachers and Pupils towards Use of Corporal Punishment in Nakuru Municipality Primary Schools, Kenya (Unpublished Master's thesis). Uni. of Kwazulu-Natal, South Africa. Available: http://hdl.handle.net

Smit EM (2010). The Role of School Discipline in Combating Violence in Schools in the East London Region (Unpublished Master's thesis). Uni. Fort Hare.

Smith K (2008). Learning Styles and Students Perception of Teachers Attitudes Among African American Students in Secondary Edu. (Unpublished Doctoral dissertation). Louisiana State Uni. Louisiana. Strong T (2003). Getting Curious About Meaning Making in Counseling. 
British J. Guidance and Counseling 31(3), 259-273.

Thompson C (2002). School crisis of Discipline. The Citizen, p.6. April 24

Ukeje BO, Akabogu GC (1992). Educational Administration. Enugu: Fourth Dimension Publishing Co.

Van der Westhuizen PC (1999). Effective Educational Management. Pretoria: Haum

Waigwa JM (1996). An Investigation into the causes of Indiscipline: The Case of Gathangi Secondary School. (Unpublished P.G.D.E. Research Project). Kenyatta University, Kenya.

Wairuri C (2004).Teachers Attitude towards the Withdrawal of Corporal Punishment in Public secondary schools in Kikuyu Division (Unpublished Master's thesis).Moi University, Kenya.

Wangari LK (2006).Effectiveness of Guidance and Counseling Program in Combating Indiscipline in Secondary Schools: A case of Nakuru Municipality, Kenya (Unpublished Master's thesis). Egerton University, Kenya.
Wango GM (2006). Policy and Practices in Guidance and Counseling in Secondary Schools in Kenya (Unpublished Doctor of Philosophy thesis). Birmingham Uni.

Yahaya, A. (2009). Disciplinary Problems among Secondary School Students in Johor Bahru, Malaysia. European J. Sci, 4(2), 56-78.

Zulu BM, Urbani G, Van der Merwe A, Van der Walt JL (2004). Violence as an impediment to a culture of teaching and learning in some South African Schools. South African J. of edu. 24(2), 170175. 